\title{
CONTRIBUCIONES DE LOS USOS DIDÁCTICOS DE LAS TIC PARA LA FORMACIÓN LITERARIA DE JÓVENES LECTORES
}

\section{ICT TEACHING USES CONTRIBUTIONS FOR THE LITERARY EDUCATION OF YOUNG READER}

\author{
Carolina González Ramírez
}

\begin{abstract}
Resumen
La enseñanza de la Literatura en la escuela es un tema que requiere atención en nuestro país. Los resultados obtenidos por los jóvenes que han rendido pruebas para la medición estandarizada de los niveles de lectura tanto nacionales (SIMCE y PSU), como internacionales (PISA), dan cuenta de que los resultados alcanzados por los jóvenes en etapa escolar en Chile son deficientes, lo que hace evidente las dificultades que los adolescentes presentan para desarrollar prácticas de lectura, tanto académicas como vernáculas. Por ello en este trabajo proponemos revisar algunas de las directrices curriculares existentes para la enseñanza de la Literatura en el contexto escolar chileno, considerando principalmente las alternativas que las TIC presentan para apoyar la labor de los docentes que han de asumir el rol de mediadores de lectura literaria en el aula.
\end{abstract}

\section{Palabras Clave}

Educación literaria, literatura, TIC, lectores en formación, prácticas lectoras 


\section{Abstract}

The teaching of Literature in school is an issue that deserves special attention in our country, Chile. The results obtained by school-age children in national tests (SIMCE and PSU) and international ones (PISA) to measure standardized reading levels are deficient, which makes evident the difficulties that adolescents have to develop both academic and vernacular reading practices. Therefore, in this paper we propose to review some of the existing curricular guidelines for the teaching of literature in the Chilean school context, mainly considering the alternatives that ICT can provide to support the work of teachers as mediators of literary reading in the classroom.

\section{Key Words}

Literary education, literature, ICT, trainee readers, reading practices

\section{Introducción}

El estudio de los cuestionarios sobre lectura que se aplican junto con PISA, llevado a cabo por la OCDE el año 2011 en Chile, vino a constatar que dos terceras partes de los estudiantes encuestados informaron que leen diariamente por placer. En dicho estudio, se señala, además, que el leer por placer está mayormente asociado a las mujeres y a estudiantes aventajados socioeconómicamente, quienes obtienen mejores resultados en las evaluaciones de comprensión, información que coincide con los datos emanados de las pruebas SIMCE aplicadas en nuestro país. Por tanto, debería ser labor de la escuela tratar de acercar la literatura a los adolescentes de todos los niveles socioeconómicos, tarea que recae en los docentes, quienes debiesen tener bastos conocimientos sobre literatura tanto canónica como del corpus de Literatura Infantil y Juvenil, así como también manejo de estrategias, recursos didácticos y herramientas digitales que les posibiliten contribuir al desarrollo de la habilidad de comprensión e interpretación de obras literarias en sus estudiantes.

Sabido es que uno de los elementos clave para el desarrollo de la comprensión es la consolidación del hábito lector. Para ello es necesario presentar a los lectores en formación un corpus de lecturas amplio y diversificado de manera que tengan opciones entre las cuales poder escoger qué leer y se despierte en ellos el interés y gusto por la lectura (Colomer, 1996, 
2005, 2015; Manresa, 2009; Margallo, 2012a). No obstante, se hace imperioso que esas lecturas que se presenten a los alumnos constituyan un desafío lector, es decir, conviene establecer un corpus de lecturas que contemple niveles de comprensión organizados de manera progresiva. Por tales razones, es que los docentes de literatura deben tener conocimiento de un gran número de obras tal como señala Chambers (1997), puesto que es necesario compaginar el canon literario obligatorio con aquellas obras que más se ajusten a los intereses de nuestros estudiantes, por lo que también es importante conocer las preferencias y gustos de los alumnos que se encuentran en nuestras aulas, así como también los saberes que estos poseen, de manera que los contenidos que el docente entregue sean pertinentes y contribuyan a que el alumno desarrolle de manera óptima las tareas de lectura, ya que contará con las herramientas necesarias para ello.

En este marco, la Literatura Infantil y Juvenil (LIJ) y el uso didáctico de TIC para enseñar literatura, juegan un papel fundamental, pues estudios realizados en este ámbito (González, 2016; González, 2014; González y Margallo, 2013; Zayas, 2009) dan cuenta de que a partir de estos dos grandes bloques de recursos es posible acercar a los estudiantes a la lectura y junto con ello, se abrirá la posibilidad de ir desarrollando habilidades interpretativas de gran complejidad a partir de la implementación de diferentes estrategias de lectura guiada de manera que con el pasar de los años los alumnos se conviertan en lectores ávidos y capaces de comprender e interpretar diferentes lecturas. De acuerdo a lo anterior, es que el objetivo de este trabajo es revisar algunas de las directrices curriculares existentes en el área de Lengua y Literatura del curriculum chileno, considerando principalmente las alternativas que las TIC presentan para apoyar la labor de los docentes que han de ejercer el rol de mediadores de lectura en aula a fin de desarrollar el gusto por la lectura en sus estudiantes.

\section{Formación de jóvenes lectores en la escuela}

De acuerdo con el paradigma de educación literaria, el mediador es quien ha de generar y moderar espacios y prácticas de lectura para aquellos lectores que se están formando, por tanto, si trasladamos esta idea a la escuela, veremos que la mediación es una labor del docente dentro del aula, pues es quien ha de procurar experimentar la lectura con los alumnos, por ejemplo: dedicar un tiempo de la clase a leer con los estudiantes, o brindarles la opción de conocer y escuchar autores, asistir a presentaciones de obras literarias o simplemente 
recomendarles un buen libro (Colomer, 2001, 2005; Margallo, 2012a; Caro, 2015). De esta forma lograremos que los lectores en formación se familiaricen con el circuito social del libro y se consoliden como lectores.

Siguiendo las premisas anteriormente expuestas, en las Bases Curriculares para Lengua y Literatura (2013) se declara que "una de las preocupaciones fundamentales de la asignatura es formar lectores habituales que acudan a la literatura como fuente de satisfacción personal, de reflexión y de conocimientos" (p.33). Sabido es que la literatura tiene una especial relevancia en la etapa en que se encuentran los estudiantes, ya que al leer, se relacionan con la obra, con ellos mismos, con la sociedad y con el lenguaje.

En función de lo anterior, en el documento se propone la siguiente interrogante ¿cómo debe ser la clase de literatura? Para ello se considera la idea de que el estudio de la literatura supone e implica leer y comentar las obras en clases, de manera que se oriente hacia la interpretación. Considerando esta idea, retomamos los señalado inicialmente, que es que el docente debe ejercer con propiedad el rol de mediador de la lectura (Chambers, 2008; Munita y Manresa, 2012), de forma que genere instancias en la clase en donde los alumnos tengan la posibilidad de formular hipótesis de lectura, discutan y reflexionen sobre sus propias interpretaciones, así como también adquieran conocimientos sobre el hecho literario (Bombini, 2006, 2008; Cuesta, 2006; Mendoza, 2004).

Resulta fundamental, entonces, que los docentes pongan en práctica estrategias que les permitan dinamizar los espacios y actividades entorno a la lectura de las obras, de manera que dicha tarea sea atractiva y motivadora para los alumnos del sistema educativo, así como también les permita adquirir los conocimientos fundamentales para develar el significado de las obras y construir una interpretación de estas, habilidad que debe ser desarrollada para alcanzar un nivel óptimo de comprensión, abordando de manera operativa y significativa el plan lector, que no solo se ha de considerar como la lista de obras para lectura domiciliaria, sino el corpus que facilitará la consolidación del hábito lector (De Vicente-Yagüe, 2015).

\section{La motivación por la lectura}

Una de las tareas que implica la lectura dice relación con la motivación. De acuerdo con la Agencia de la calidad de la educación (2016) "la motivación por la lectura es entendida como el interés del estudiante por la lectura, el disfrute de los espacios destinados a ella (no visto como 
una actividad obligatoria) y la valoración positiva del debate en torno a las lecturas y los libros" (p.19). Generar interés tiene relación con establecer espacios y momentos para la lectura (Colomer, 2005; Solé, 1995). Sin embargo, sabemos que en nuestro sistema educativo los tiempos son escasos y los docentes deben abordar diversos contenidos del currículum, los que se han establecido como el foco de la clase, razón por la cual la literatura pasa a un segundo plano y se suele abordar de manera instrumental y alejada del contexto de aula como ocurre habitualmente con las lecturas programadas en el plan lector, lo que finalmente redunda en un desinterés por las tareas de lectura encomendadas a los alumnos, ya que se ven enfrentados a un ejercicio obligatorio con el que deben cumplir, sin tener un proceso de acompañamiento que les entregue claves y estrategias para incrementar y mejorar sus prácticas lectoras.

Resulta entonces necesario clarificar qué se entiende por lector, puesto que es lo que nos permitirá determinar las características de su formación. Considerando que en los últimos años ha surgido una literatura infantil y juvenil de calidad, el lector de esta literatura ha sido definido por Colomer (1998) inicialmente como:

"un lector niño, niña o adolescente que aprende socialmente y a quien se dirigen textos que intentan favorecer su educación social a través de una propuesta de valores, de modelos de relación social y de interpretación ordenada del mundo. $Y$, en segundo lugar, como un lector infantil o adolescente que aprende una forma cultural codificada-la literatura-, y a quien se dirigen unos textos que traspasan unas formas fijadas del imaginario, unas formas narrativas con tradición de uso literario, una valoración estética del mundo y un uso especial del lenguaje" (p.144).

En esta propuesta, el niño o adolescente lector se forma en un entorno social, aprendiendo a partir de las lecturas, las convenciones sociales que le permiten interpretar el mundo. Es por esta razón, que es necesario hacer un trabajo formativo en el que se guíe al lector en formación a familiarizarse con el lenguaje literario de manera que se vaya alfabetizando en prácticas de lectura a fin de que pueda asegurarse la integración paulatina a comunidades lectoras. En el marco del modelo de educación literaria, el lector es considerado como un receptor activo que debe poner en práctica diversas habilidades cognitivas y también sociales para interpretar un texto literario. Para ello el rol del lector y el dominio de habilidades lectoras es fundamental. 
De acuerdo con Cairney (1992), la tarea del lector consiste en tratar de aproximarse a un lector ideal, es decir, a alguien que asimila el significado que el escritor ha procurado comunicar, por tanto, en el proceso de formación y desarrollo de habilidades de lectura la mediación que el docente realiza es un factor decisivo; sin embargo, algunas investigaciones indican que este rol no ha sido del todo explotado por los docentes tanto por desconocimiento de estrategias de mediación así como también por la debilidades evidenciadas en su formación literaria.

Ahora bien, el lector, como agente activo, debe realizar un ejercicio de lectura literaria que supone la adquisición y desarrollo de habilidades interpretativas, es decir, una competencia literaria que le permita enfrentarse a las lecturas que se le proponen. La competencia literaria es definida por Lomas (2003) como la "adquisición de hábitos de lectura, capacidad para disfrutar y comprender distintos textos literarios y el conocimiento de algunas obras y autores representativos de la historia de la literatura" (p.5).

Es por esta razón que es necesario que los docentes trabajen en la creación de espacios y tiempos para la lectura, así nuestros alumnos podrán establecer una conexión personal con el texto y desarrollar habilidades interpretativas que les permitan realizar lecturas de nivel experto. De esta manera, la escuela estará contribuyendo a la formación de lectores literarios competentes y es lo que nos interesa saber, si efectivamente estas acciones se están realizando, dado que durante los últimos años, con el propósito de disminuir la brecha existente entre los sectores desfavorecidos económicamente de aquellos que no lo son, se han propuesto diversas iniciativas para el fomento de la lectura en nuestro país, como por el ejemplo el Plan Nacional de Lectura 2015-2020 impulsado por el Ministerio de Educación, en conjunto con otras entidades gubernamentales. Los ajustes curriculares (2013; 2015), documentos en los que se sugiere la incorporación de nuevos títulos de obras literarias correspondientes al corpus LIJ, recursos digitales para el acompañamiento de la lectura, y actividades de motivación a la lectura, lo que deja entrever que se está poniendo atención a las prácticas de lectura vernáculas de los alumnos, así como también a sus intereses y necesidades. En el siguiente apartado abordaremos las posibilidades didácticas que las TIC presentan para motivar y mediar la lectura de obras literarias. 


\section{Usos didácticos de las TIC para formar jóvenes lectores}

Considerando el contexto actual de educación en nuestro país, partiendo por los alumnos que están en las aulas, los docentes que día a día trabajan con ellos y los centros educativos que los acogen, tenemos un panorama diverso, pero a la vez homogéneo en cuanto a necesidades e intereses que de cierto modo es posible organizarlos en criterios generacionales ¿Qué ha pasado? Los docentes han debido darse a la tarea de conocer los intereses de sus estudiantes con el propósito de poder acercar los conocimientos que ellos les han de entregar a su propia realidad, es decir, "zona de desarrollo próximo", concepto acuñado por Vigotsky en la década del '30. Considerando este aspecto fundamental en la formación de un estudiante conseguiremos un aprendizaje realmente significativo.

En este proceso de formación, que en la actualidad es bidireccional, pues el docente ha de formar a sus alumnos, pero sus alumnos también lo forman a él, pues día a día el profesor aprende nuevas cosas que en el mundo de los niños y jóvenes son habituales, pero como adultos muchas veces desconocemos, más aún aquello que tiene que ver con tecnologías de la información y la comunicación (TIC), pues estas avanzan en su desarrollo y cambian rápidamente, sin dar tregua.

En esta vorágine de constante cambio, es que como docentes debemos considerar su utilización para mediar procesos de enseñanza y aprendizaje, puesto que ya el contexto mismo nos evidencia que es necesario utilizarlas, ya que es algo que nuestros estudiantes dominan y porque nuestro currículum para Lengua y Literatura (2013; 2015) así lo exige. Desde el área que nos compete, literatura, específicamente, educación literaria, tenemos que las TIC presentan una serie de posibilidades para enseñar literatura. González y Margallo (2013), proponen una metodología de usos de las TIC para la educación literaria que se articula en tres bloques: TIC para la información, TIC para la socialización y TIC para la creación.

De acuerdo a esta propuesta teórica, las TIC para la información corresponden a todas aquellas herramientas que permitan al estudiante familiarizarse con el hecho literario, es decir, investigar para contextualizar, por ello se consideran aquí bases de datos, bibliotecas digitales, revistas especializadas en literatura, entre otras. En cuanto a las TIC para la socialización, se sostiene que las aplicaciones digitales, especialmente aquellas que permiten interacción con 
otros usuarios en red como las redes sociales, blogs, chats, plataformas de publicación como Youtube, Instagram, entre otras, dan la posibilidad de que el lector pueda compartir su lectura e interpretación de obras con otros lectores y de esta manera formar un círculo de lectura, recreando la antigua figura de las tertulias literarias. Finalmente, las TIC para la creación, se constituyen como el espacio en el que el joven lector y escritor puede realizar sus propios textos y darlos a conocer; desde el ámbito estrictamente educativo tenemos las wikies, sitiesgoogle, google docs, entre otros, pero desde las prácticas de lectura y escritura en red de tipo vernáculas (Cassany, 2009; 2012; Aliagas, 2011), tenemos el fancfiction, práctica de escritura vernácula que ha tomado fuerza y se ha comenzado a hacer cada vez más masiva y conocida entre los adolescentes, quienes de forma anónima o bajo un seudónimo pueden compartir sus producciones literarias en línea.

La tarea que nos queda a nosotros como docentes de Lengua y Literatura es lograr aunar estos dos componentes, literatura y TIC de manera que podamos generar instancias en las que nuestros alumnos se sientan llamados a participar, pues como vimos anteriormente, un componente esencial para el fomento del hábito lector y la consolidación de este es la motivación, que en un punto ha de generarla el maestro, pero luego ha de ser intrínseca al estudiante, pues es él quien debe decidir finalmente qué leer y qué no.

\subsection{Inclusión metodológica de las TIC en la clase de Literatura}

En relación a lo expuesto en el apartado anterior, es posible señalar que las posibilidades de inclusión y uso de TIC para la enseñanza de la literatura en el aula son inagotables, pues el número de opciones metodológicas es tan amplio como recursos y aplicaciones digitales hayan.

Para el ámbito educativo, el mayor aporte de la web 2.0 ( Adell, 2010) ha sido la generación de espacios virtuales e interactivos para que los estudiantes puedan aprender y en nuestro caso, a partir de la literatura, puedan interpretar y crear sus propias obras. Este es uno de los aportes más relevantes de la red para la implementación del modelo de educación literaria en el marco de la sociedad de la información. 
Así el estudio, análisis y generación de materiales didácticos que incorporen TIC para el tratamiento de la literatura permite obtener una visión general de las potencialidades que las TIC entregan para el logro de los objetivos de educación literaria, tanto lo que se refiere a avance en la interpretación como el fomento del hábito lector (Margallo, 2012a). No obstante, es necesario poner atención a la calidad de los materiales didácticos diseñados para tales efectos, de manera que se logre tener claro el foco que es la literatura y no el recurso digital propiamente tal; por ejemplo, es importante que el conocer y aprender a usar determinados recursos no entorpezca el trabajo que se debe realizar entorno al hecho literario.

En cuanto a las TIC para la información, podemos señalar concretamente que los docentes han de gestionar el acceso a las fuentes de información, es decir, cautelar ya sea a partir de búsquedas guiadas en la red, webquest, o cazas del tesoro los sitios que los estudiantes han de revisar para poder extraer información que sea relevante y consistente para enriquecer su conocimiento respecto del hecho literario que se le ha encomendado estudiar.

Las TIC para la socialización en tanto, contemplan la socialización de experiencias en torno a la lectura literaria por parte de los estudiantes y también pueden participar los docentes. Por ejemplo, resulta de gran interés para los alumnos ver a sus docentes leyendo o recomendando una obra y una de las vías más rápida para hacerlo es la utilización de redes sociales. Así, docentes y alumnos pueden crear sus propios booktubers, booktrailers, o recomendaciones literarias a través de podscast, códigos QR, entre otras. Estas experiencias pueden decantar en clubes de lectura, que funcionan on-line y así se consolidan prácticas de socialización literaria y el compartir con otros la experiencia en torno la lectura se materializa.

Finalmente, las TIC para la creación, pueden ser un soporte para abordar la escritura creativa en el aula y lograr que los estudiantes generen sus propios textos con intención literaria. De este modo, pasarán de lectores-usuarios a ser creadores de textos literarios y podrán gestionarlos a su gusto en internet. Ya mencionábamos en el apartado anterior el fanfiction, género que durante los últimos 5 años se comenzó a hacer más masivo entre los adolescentes, sin embargo, revisando las novedades y actualizando lo que la web ofrece, encontramos la plataforma de Wattpad, una plataforma que se promociona como el mejor lugar para leer y compartir historias. Para acceder a ella es suficiente con registrarse y comenzar a subir las propias publicaciones o leer lo que otros usuarios han compartido anteriormente. 
Otras alternativas que se pueden considerar para que los alumnos experimenten la literatura a partir de la escritura creativa son: póster digitales, podcast, blogs, reinterpretaciones de obras en formato video, reediciones digitales de obras, lo que les permiten abordar los textos literarios desde una visión reflexiva y analítica, a la vez que se fomenta su capacidad creativa.

\section{Comentarios finales}

Rescatando la idea inicial de la que la literatura tiene una función social y esta va cambiando a medida que las sociedades evolucionan, el panorama que hemos descrito es un fiel reflejo de esta idea. La literatura es sin duda un medio para comunicarnos, para expresarnos, para llegar al otro y en el mundo vertiginoso que estamos viviendo las TIC juegan un rol fundamental, pues son la clave para acceder a un sin fin de información, solo necesitamos aprender a decodificarla. Es por ello, que la Escuela debe incorporar estos recursos a los procesos de enseñanza y aprendizaje, pues se ha visto que funcionan como catalizadores de la motivación de los alumnos por realizar tareas escolares.

Asimismo, creemos que es necesario destacar la importancia de incentivar a los estudiantes a llevar a cabo prácticas de lectura alternativas y ampliar su corpus de lecturas (Díaz-Plaja, 2009). No solo debemos pensar en realizar el control de lectura mensual correspondiente al plan lector o contextualizar y comentar la obra antes de la prueba, sino más bien, aventurarnos en la tarea de proponer proyectos literarios (Camps, 1994; Margallo, 2012b) a través de los cuales dar forma a sus respuestas creativas a la lectura (Margallo, 2013), además de poner en práctica otras habilidades que contribuyan a su formación como lectores competentes, que no solo corresponde a la comprensión lectora, sino también a la producción tanto oral como escrita.

Por ello, es necesario trabajar con las TIC de manera didáctica y no solo instrumental, esto es, generar una metodología que permita proponer actividades en las que estos recursos estén presentes, aprovechando sus potencialidades las que trascienden la simple transmisión de información, sino que aportan o contribuyen a la generación de conocimiento a partir de la literatura.

En definitiva, nosotros los docentes o también podríamos autodenominarnos mediadores literarios debemos considerar como un desafío el generar espacios para la reflexión antes, 
durante y después de la lectura literaria de los estudiantes, y junto con ello, también darles la posibilidad de exponer su lectura a través de otras modalidades que no sean siempre el examen o control de lectura o una interrogación oral, sino que se les dé la oportunidad para que a través del uso diversas herramientas TIC y recursos digitales desarrollen sus hipótesis e interpretaciones de lectura y finalmente se genere el gusto por la lectura literaria, materializando en la escuela el contagio por la lectura (Trujillo, 2010).

\section{Bibliografía}

Adell, J. (2010). Educación 2.0. En. Barba, C; Capella, S. (coords.). Ordenadores en las aulas. La clave es la metodología. Barcelona: Graó, pp.19-33.

Aliagas, C. (2011). "Llegir en temps de lleure. Estudi de cas d'una colla d'adolescents amb el virus de la desafecció lectora". Articles de Didàctica de la Llengua i de la Literatura 53, Monogràfic: Què llegeixen els “no lectors”?, 45-57.

Bombini, G. (2006). Reinventar la enseñanza de la lengua y la literatura. Buenos Aires, Argentina: Libros del Zorzal.

Bombini, G. (2008). La lectura como política educativa. Revista Iberoamericana de Educación. № 46, 19-35.

Cairney. T. H. (1992). Enseñanza de la comprensión lectora. Madrid: Ministerio de Educación y Ciencia: Morata.

Camps, A. (1994). "Proyectes de llengua entre la teoria i la pràctica". Articles de Didàctica de la Llengua i la Literatura, (2), 7-20.

Caro, M. T. (2015). Fundamentación científica de la Educación Literaria. En: Guerrero, P y Caro, M.T. (coords.). Didáctica de la lengua y la educación literaria. Madrid: Pirámide, 261-288.

Cassany, D. (2009). Prácticas literarias vernáculas de adolescentes y jóvenes: del cánon a la calle. En $X$ Congreso latinoamericano de Lectura y Escritura. Lima, Perú.

Cassany, D. (2012). En-línea. Leer y escribir en la red. Barcelona: Anagrama.

Colomer, T. (1996). La didáctica de la literatura: temas y líneas de investigación e innovación. En Lomas, C. (coord.). La educación lingüística y literaria en la enseñanza secundaria. Barcelona: Horsori,123-142.

Colomer, T. (1998). Introducción a la literatura infantil y juvenil. Madrid: Síntesis. 
Colomer, T. (2001): La enseñanza de la literatura como construcción de sentido. Lectura y vida. Revista Latinoamericana de lectura. Asociación Internacional de Lectura (IRA), Año XXII, nำ1, 6-23.

Colomer, T. (2005). Andar entre libros. México: Fondo de cultura económica.

Colomer, T. (2015). Introducción a la literatura infantil y juvenil actual. Madrid: Síntesis

Cuesta, C. (2006). Discutir sentidos: La lectura literaria en la escuela. Buenos Aires: Libros del Zorzal.

Chambers, A. (1997). Cómo formar lectores. [En línea]. Disponible en: http://www.lablaa.org/blaavirtual/ninos/sitio_lectura/noviembre/educadores.htm

Chambers, A. (2008). Dime: Los niños, la lectura y la conversación. México: Fondo de cultura económica.

De Vicente-Yagüe. M.T. (2015). "Fomento de la lectura: el plan lector. El plan formativo en la educación lectoliteraria”. En: Guerrero, P y Caro, M.T. (coords.). Didáctica de la lengua y la educación literaria. Madrid: Pirámide, 297-303.

Díaz-Plaja, A. (2009). Entre libros: la construcción de un itinerario lector propio en la adolescencia. En: Colomer, T. (coords.) Lecturas adolescentes. Barcelona: Graó.

González, C. y Margallo, A. M. (2013). Usos didácticos de las TIC para la formación de lectores en vías de la educación literaria. Revista Foro Educacional, 22, UCSH, Santiago de Chile, 31-51.

González, C. (2016). "Tecnologías de la información y la comunicación y educación literaria en la formación inicial del profesorado". Tesis para optar al grado de Doctor en Didáctica de la Lengua y la Literatura, Universidad Autónoma de Barcelona, Barcelona, España. En línea. http://hdl.handle.net/10803/369580

Lomas, C. (2003). La educación literaria en la enseñanza obligatoria. Revista Kikiriki, 64 [en línea]. Disponible en: http://www.lenguaweb.info/didactica-de-la-literatura/470-laeducacion-literaria-en-la-ensenanza-obligatoria.

Manresa, M. (2009). Lecturas juveniles: el hábito lector dentro y fuera de las aulas. Textos, Barcelona: Graó, 44-54.

Margallo, A. M. (2012a):"Claves para formar lectores adolescentes con talento".Leer.es. [en línea]. Disponible en: http://docentes.leer.es/2012/03/13/claves-para-formar-lectoresadolescentes-con-talento-ana-maria-margallo/ 
Margallo, A. M. (2012b): "La educación literaria como eje de la programación". En: Ruíz Bikandi, U. (coord.). Didáctica de la lengua castellana y la literatura. Barcelona: Graó, 167186

Margallo, A.M. (2013): "Com elaborar la resposta lectora als textos literaris mitjant l'escriptura". Articles de Didàctica de la Llengua i la Literatura, núm., 61, pp. 65-71.

Mendoza, A. (2004). La educación literaria: bases para la competencia lecto-literaria. Málaga, Aljibe.

Mineduc (2013; 2015). Bases curriculares. Lengua y Literatura. Santiago de Chile: Gobierno de Chile.

Ministerio Ministerio de las Culturas, las Artes y el Patrimonio (2016). Plan Nacional de la Lectura 2015-2020. Gobierno de Chile. http://plandelectura.gob.cl/wpcontent/uploads/2016/12/Plan-Nacional-Lectura-web-6-12-2016.pdf

Munita, F. y Manresa, M. (2012). “La mediación literaria”. En: Colomer, T. y Fitipaldi, M. (Coords.). La literatura que acoge: Inmigración y lectura de álbumes. Venezuela: Banco del libro.

Trujillo, F. (2010). Prácticas de lectura literaria en las aulas de secundaria. Lectura y Vida. Recuperado de: http://www.lecturayvida.org.ar

Zayas, F. (2009). "Escribir y leer en la Red: nuevas prácticas discursivas". En Lara, T; Zayas, F., Arrurero, N., y Larequi, E. La competencia digital en el área de Lengua. Barcelona: Octaedro, 39-68.

\section{REFERENCIA BIBLIOGRÁFICA}

González, C: contribuciones de los usos Didácticos de las TIC para la formación literaria de jóvenes lectores Aula de Encuentro, no2 (20), pp. 21-34. DOI: https://dx.doi.org/10.17561/ae.v20i2.2 
Carolina González Ramírez es Profesora del Dpto. de Didáctica y Prácticas del ILCL de la Pontificia Universidad Católica de Valparaíso Correo-e: carolina.gonzalez.r@pucv.cl

Enviado: 6 de mayo de 2018 Aceptado: 14 de agosto de 2018 